\title{
ROBUST CONTROL DESIGN PROCEDURE BASED ON PARTICLE SWARM OPTIMIZATION AND KHARITONOV'S THEOREM WITH AN APPLICATION FOR PMSMs
}

\author{
Lucas Cielo Borin ${ }^{1}$, Caio Ruviaro Dantas Osório ${ }^{1}$, Gustavo Guilherme Koch ${ }^{1}$, Thieli Smidt Gabbi ${ }^{2}$ \\ Ricardo Coração de Leão Fontoura de Oliveira ${ }^{3}$ and Vinícius Foletto Montagner ${ }^{1}$ \\ ${ }^{1}$ Universidade Federal de Santa Maria (UFSM) - Santa Maria, RS, Brazil \\ ${ }^{2}$ Universidade Federal do Rio Grande do Sul (UFRS) - Porto Alegre, RS, Brazil \\ ${ }^{3}$ Universidade de Campinas (UNICAMP) - Campinas, SP, Brazil \\ e-mail: (lukascielo, caio.osorio, gustavoguilhermekoch, thielisgabbi,vfmontagner)@gmail.com, ricfow@dt.fee.unicamp.br
}

\begin{abstract}
This paper proposes an automatic procedure for robust control design applicable to power converters based on particle swarm optimization and Kharitonov's Theorem. The main benefit is to provide control gains that have a theoretical certificate of robust stability and also accomplish multiple performance criteria in a design less dependent of human-machine interaction. Regarding the particle swarm optimization, each particle represents a controller candidate whose performance is evaluated by means of an objective function, using the vertices of a polytopic model of the plant and the four polynomials of Kharitonov's Theorem. The effectiveness of the proposed procedure is illustrated by means of a case study that considers the speed control of a permanent magnet synchronous motor subject to uncertain mechanical and electrical parameters. The designed controllers, obtained in an offline way, yield good trade-offs between performance and robustness, as confirmed by simulation and experimental evaluations. Analyses show superior results with the proposed strategy compared to a genetic algorithm and to a design tool specialized for PID tuning, indicating its viability as an alternative for robust control design in power electronics.
\end{abstract}

Keywords - Kharitonov's theorem, Particle swarm optimization, Permanent magnet synchronous motors, Power converters, Robust control.

\section{INTRODUCTION}

A common point in designing controllers for engineering problems, particularly for control of power converters, is the need to take into account trade-offs among different objectives, such as fast dynamic responses, accurate reference tracking, good disturbance rejection, control action limitation, robustness against parametric uncertainties, etc [1]-[4]. An alternative to deal with design problems with multiple objectives and constraints that can be difficult to be expressed analytically is to use intelligent algorithms, which can evolve based on the evaluation of numerical simulations or experimental data [5]. Among well-known intelligent algorithms, it is possible to highlight the particle swarm

Manuscript received 02/27/2020; first revision 05/30/2020; accepted for publication 06/26/2020, by recommendation of Editor Demercil de Souza Oliveira Jr. http://dx.doi.org/10.18618/REP.2020.2.0008 optimization (PSO), proposed in [6], for reasons such as the ability to evolve without relying on the objective function derivative, the avoidance of local minima, the fast execution time and simple computational implementation [7]. Since its proposition, this algorithm has been used for optimization in several applications, as in [8]. However, as pointed out by [9], concerning control tuning for power converters, few works with metaheuristics, including PSO, have been produced, indicating a potential for further investigation with this technique.

In the context of PSO applied to tune fixed gain controllers for power converters, this strategy was used, for instance, in [7], [10]-[13], mainly to design PI and PID controllers. The optimization by PSO is guided by an objective function. For instance, [10]-[12] aim on time domain specifications, while [7], [13] aim on frequency domain specifications. A common point in these works is not exploring simultaneously time and frequency domain criteria for tuning controllers. Although there exist correlation between specifications in these domains (e.g., between overshoot and phase margin or between steady state error and low frequency gain), these correlations are based, for instance, in approximating the plant as second order models, and may lead to inaccurate results. Therefore, exploring time and frequency domain specifications simultaneously can be interesting but, on the other hand, the task becomes more challenging when the system is subject to uncertain parameters and to limits in the control action. These points are investigated here, incorporating multiple practical constraints and robustness against uncertain parameters during the controller design stage, not requiring exhaustive analyses after the design to certify robustness and performance.

Assuming that the plant is described by a model whose coefficients are not precisely known, but belong to real intervals, the robust stability under uncertain parameters can be certified by the Kharitonov's Theorem [4], [14], [15]. In the context of power electronics, Kharitonov's Theorem was used to certify the robustness against uncertainties, for instance, in Buck converters [16], microgrids [17]-[19], and PMSMs [20]-[22]. Although Kharitonov's Theorem has been applied to define, a priori, regions of robust stability, employed for choosing the control gains, the use of this tool during the control tuning stage still demands further investigation. Then, to the best of the authors' knowledge, there is a lack of works with Kharitonov's Theorem combined with PSO strategy 
applied in robust control design procedures for controllers of power converters.

The main contribution of the present work is an automatic robust control design procedure that combines PSO and Kharitonov's Theorem to cope with multiple practical design constraints and robustness against parametric uncertainties, providing control gains that ensure suitable results for power converters. As performance criteria, the proposed procedure encompasses the deviation from reference values for phase margin and crossover frequency and limits for gain margin, overshoot, steady state error and control signal saturation. To make the procedure computationally viable, these performance criteria are evaluated at the vertices of a polytopic model of the plant, which is a necessary condition to assure performance for the whole polytope. The robust stability for the entire domain of the uncertain parameters is certified by Kharitonov's Theorem, which is a sufficient condition that can be tested in a fast way by evaluating the stability of only four polynomials. This last feature makes the proposed procedure novel with respect to previous works dealing with PSO for control tuning in power converters, since the synthesized controllers have a theoretical certificate of robust stability for closed-loop systems.

The procedure, which is summarized in Section III-D, is implemented offline, leading to simple fixed gain controllers that can be applied for a large class of power converters affected by interval parameters. To exemplify, a case study is presented, focusing on the speed control of a permanent magnet synchronous motor (PMSM) with uncertain electrical and mechanical parameters. Differently from previous works which apply PSO or Kharitonov's Theorem to PMSMs, as for instance, [20]-[23], here multiple design criteria are employed to guide the PSO to find fixed control gains ensuring operation under limited control signal, with a theoretical certificate of stability under uncertain plant parameters being provided for the designed gains. Experimental results are provided, illustrating suitable responses, with superior performance of the proposed design procedure when compared to other control design alternatives.

\section{PROBLEM DESCRIPTION}

Consider the plant transfer function

$$
G(s)=\frac{f_{0}(p)+f_{1}(p) s+f_{2}(p) s^{2}+\cdots+f_{n}(p) s^{n}}{g_{0}(p)+g_{1}(p) s+g_{2}(p) s^{2}+\cdots+g_{n}(p) s^{n}} .
$$

where the coefficients are function of uncertain physical parameters, which are given by

$$
p:=\left[p_{1}, p_{2}, \cdots, p_{L}\right], p_{\ell} \in\left[p_{\ell}{ }^{-}, p_{\ell}{ }^{+}\right], \ell=1 \ldots L .
$$

This type of representation is suitable for averaging model of power converters where the physical parameters (e.g., inductances, capacitances and resistances) are not precisely known, but lie on known intervals [14].

In order to ensure stability and performance over the entire domain of uncertain parameters, assume a controller

$$
G_{c}(s)=\frac{x_{0}+x_{1} s+x_{2} s^{2}+\cdots+x_{m} s^{m}}{y_{0}+y_{1} s+y_{2} s^{2}+\cdots+y_{m} s^{m}} .
$$

where the controller coefficients are defined by the vector

$$
c=\left[x_{0}, x_{1}, \cdots, x_{m}, y_{0}, y_{1}, \cdots, y_{m}\right] .
$$

The controller transfer function (3) is composed by fixed coefficients to be computed offline. For example, for a PI controller, $x_{0}=K_{I}, x_{1}=K_{P}, y_{0}=0, y_{1}=1$, and $c=$ $\left[K_{I}, K_{P}, 0,1\right]$.

\section{A. Robust Stability based on Kharitonov's Theorem}

The transfer function $G(s)$ in (1) can be represented by the interval family of plants of degree $n$ given by

$$
G_{k}(s)=\frac{a_{0}+a_{1} s+a_{2} s^{2}+\cdots+a_{n} s^{n}}{b_{0}+b_{1} s+b_{2} s^{2}+\cdots+b_{n} s^{n}} .
$$

where the coefficients $a_{\ell}$ and $b_{\ell}$ are obtained from the evaluation of $f_{\ell}(p)$ and $g_{\ell}(p)$, leading to bounded real intervals given by

$$
a_{\ell} \in\left[a_{\ell}{ }^{-}, a_{\ell}{ }^{+}\right], b_{\ell} \in\left[b_{\ell}{ }^{-}, b_{\ell}{ }^{+}\right], \quad \ell=0, \cdots, n .
$$

Given the controller (3) and the interval plant (5), the characteristic polynomial $D(s)$ of the closed-loop system can be written in the interval form as

$$
D(s)=d_{0}+d_{1} s+d_{2} s^{2}+\cdots+d_{n+m} s^{n+m} .
$$

with bounded coefficients

$$
d_{\ell} \in\left[d_{\ell}^{-}, d_{\ell}^{+}\right], \quad \ell=0, \cdots, n+m
$$

According to Kharitonov's Theorem, one has that if the four polynomials

$$
\begin{aligned}
& K_{1}(s)=d_{o}{ }^{-}+d_{1}{ }^{-} s+d_{2}{ }^{+} s^{2}+d_{3}{ }^{+} s^{3}+d_{4}{ }^{-} s^{4}+\cdots, \\
& K_{2}(s)=d_{o}{ }^{-}+d_{1}{ }^{+} s+d_{2}{ }^{+} s^{2}+d_{3}{ }^{-} s^{3}+d_{4}{ }^{-} s^{4}+\cdots, \\
& K_{3}(s)=d_{o}{ }^{+}+d_{1}{ }^{-} s+d_{2}{ }^{-} s^{2}+d_{3}{ }^{+} s^{3}+d_{4}{ }^{+} s^{4}+\cdots, \\
& K_{4}(s)=d_{o}{ }^{+}+d_{1}{ }^{+} s+d_{2}{ }^{-} s^{2}+d_{3}{ }^{-} s^{3}+d_{4}{ }^{+} s^{4}+\cdots
\end{aligned}
$$

are Hurwitz, then $D(s)$ in (7) is Hurwitz and thus the closedloop system is stable for all values of the interval coefficients.

The proof can be found in [4].

Definition 1. KT stable: If (9) is satisfied in the scenario specified above, the uncertain closed-loop system will be called from now on, in this paper, as KT stable, i.e., stable based on Kharitonov's Theorem.

When dealing with plants with coefficients that are not independent, as can be the case of power converters, where the physical parameters may affect more than one coefficient of the transfer function (1) at the same time, Kharitonov's Theorem tested as in (9) is a sufficient condition for robust stability of system (1) for a given controller (3). In these cases, due to its computational simplicity, this condition is worth to be used for a fast evaluation of stability [4], [24].

\section{B. Performance Criteria based on Polytopic Representation}

In classical control design procedures for power converters, it is very common to specify performance in terms of frequency domain criteria, such as crossover frequency $\left(\omega_{c o}\right)$, phase margin (PM) and gain margin (GM) [3], [25], [26]. 
Moreover, it is also desirable to shape the closed-loop system step response based on time domain performance constraints, such as overshoot (OV) and steady state error $\left(e_{s s}\right)$. A good trade-off among these specifications is a challenge, becoming more difficult when uncertain parameters and control saturation (i.e., a limit for the amplitude of the control signal $u$ ) must be taken into account in the design.

One way to estimate the above performance measures for plants affected by uncertain parameters, as (1), for a given fixed gain controller (3), is from a polytopic representation of the plant [27]. By combining all extreme values of the physical parameters in (2), the resulting polytopic model has $V=2^{L}$ vertices, given by

$$
G(s)=\sum_{i=1}^{V} \alpha_{i} G_{i}(s), \sum_{i=1}^{V} \alpha_{i}=1, \quad \alpha_{i} \geq 0, \quad i=1, \ldots, V .
$$

where $G_{i}(s)$ are the vertices of the polytopic plant model. The measures of PM, $\omega_{c o}$, GM, OV, $e_{s s}$ and the maximum of $u$ can be carried out evaluating a set of time and frequency domain responses, only at the vertices of the polytopic model.

Notice that even though the worst case values of the above measures may not be captured by only evaluating the responses at the vertices of the polytopic plant, the proposed procedure becomes appealing from a computational point of view, leading to effective results in power electronics plants, as illustrated by the case study in this paper. Nevertheless, in order to include a theoretical certificate of robust stability valid for the entire domain of uncertainties, Kharitonov's Theorem is then aggregate to the proposed approach.

\section{Control Design Problem Definition}

The problem to be solved in this paper is to find, in an offline procedure, the control gain vector (4), such that:

- an objective function including performance specifications in terms of the time and frequency domain criteria PM, $\omega_{c o}, \mathrm{GM}, \mathrm{OV}, e_{s s}$ and the maximum of $u$ is optimized for the vertices of the polytopic model;

- the closed-loop uncertain system (1) with controller (3) is $K T$ stable.

\section{PROPOSED CONTROL DESIGN PROCEDURE}

In order to provide a systematic solution for the above problem, this section presents the definition of the objective function, the search space for the gains, the PSO algorithm and summarizes the proposed design procedure.

\section{A. Objective Function}

For each controller vector candidate $c$ in (4), the objective function proposed here returns a real positive scalar computed based on three terms, $\alpha(c), \beta(c)$ and $\gamma(c)$, that measure the quality of the system performance with a given controller.

The first term, $\alpha(c)$, is defined as

$$
\alpha(c)=\max _{j=1, \cdots, V}\left(\left|\frac{\mathrm{PM}^{*}-\mathrm{PM}_{j}(c)}{\mathrm{PM}^{*}}\right|+\left|\frac{\omega_{c o}{ }^{*}-\omega_{c o j}(c)}{\omega_{c o}{ }^{*}}\right|\right) .
$$

and measures the worst case deviation of the phase margin and crossover frequency provided by the controller candidate $c$, from reference values $\mathrm{PM}^{*}$ and $\omega_{c o}{ }^{*}$, computed for all vertices of the polytopic model, represented by the index $j$. Notice that the values of $\mathrm{PM}_{j}(c)$ and $\omega_{c o j}(c)$ can be easily computed by means of a specialized function, as the function margin, from MATLAB.

Note that minimizing only $\alpha(c)$ may not be sufficient to ensure good time and frequency responses. Aiming at improving the performance, a second term, $\beta(c)$, is taken into account, playing the role of a penalty factor in the objective function, being computed as

$$
\beta(c)=\left\{\begin{aligned}
& \text { if } \mathrm{GM}_{j}(c) \geq \mathrm{GM} \text { and } \mathrm{OV}_{j}(c) \leq \overline{\mathrm{OV}} \\
& 1, \quad \begin{array}{l}
\text { and }\left|e_{s s j}(c)\right| \leq \overline{e_{s s}} \text { and }\left|u_{j}(c)\right| \leq \bar{u} \\
\text { for all } j=1, \cdots, V
\end{array} \\
& 10^{6}, \quad \text { otherwise. }
\end{aligned}\right.
$$

$\beta(c)$ returns an unitary value if all the conditions in (12) are satisfied, i.e., if the controller candidate $c$ ensures, for each vertex, compliance with prescribed lower bound GM and upper bounds $\overline{\mathrm{OV}}, \overline{e_{s s}}$ and $\bar{u}$. Otherwise, $\beta(c)$ returns the value $10^{6}$, in order to penalize the objective function for this controller candidate. The indices in (12) can be easily computed, for instance, by means of the functions margin and step, from MATLAB.

Note that choosing the constraints in $\alpha(c)$ and $\beta(c)$ is not a difficult task, as typical values for these parameters can be found in the literature or can be defined based on the converter under investigation (e.g., $\mathrm{PM}$ of $60^{\circ}$, GM from 2 to 5 , etc. [3], [28]).

The third term of the proposed objective function, $\gamma(c)$, also plays the role of a penalty factor, related with the robust stability ensured by means of Kharitonov's Theorem, and is given by

$$
\gamma(c)=\left\{\begin{array}{cl}
1, & \text { if the closed-loop system is KT stable } \\
10^{6}, & \text { otherwise }
\end{array}\right.
$$

Recall that a positive evaluation of Kharitonov's Theorem is a theoretical guarantee of robust stability for the closedloop system under uncertain parameters. Notice that since the approach here is based on computational functions to obtain the measures of the time and frequency responses, and to compute the roots of the Kharitonov's polynomials, it can be applied for plant and controller models of arbitrary order, avoiding analytical formulation, which are progressively more difficult to handle as the order of the transfer functions increase.

Finally, the objective function can be expressed as

$$
f(c)=\alpha(c) \beta(c) \gamma(c) .
$$

where, to summarize: $\alpha(c)$ is related to get controllers $c$ that lead to open-loop responses with phase margins and crossover frequencies close to reference values; $\beta(c)$ is related to get controllers that comply with limitation for gain margin, overshoot, steady state error and control saturation; $\gamma(c)$ is related to get controllers that ensure the $K T$ stability of the closed-loop system.

The best controller associated to the objective function (14) is the vector $c$, given in (4), which minimizes $f(c)$ in a search 
space. Thus, the control design problem can be expressed by the optimization problem

$$
c^{\star}=\arg \min _{c \in \mathscr{C}} f(c) .
$$

where $\mathscr{C}$ is the search space defined in the next subsection.

It is worth to mention that different objective functions could be defined to guide the control design task, as those used in [7], [10], [29]. The specifications in (14) were chosen here considering traditional performance constrains used in practice in power electronics (e.g., PM, GM, $\omega_{c o}$ ). As an original aspect, (14) includes in the control design stage the robust stability assessment (i.e., KT stability) and actuator saturation evaluation. These last constraints make the design more difficult, but able to cope with real challenges of parametric uncertainties and limited control signal.

\section{B. Search Space}

The space for searching the control gains is defined here as

$$
\mathscr{C}=\left\{\begin{array}{l}
\left(x_{0}, x_{1}, \cdots, x_{m}, y_{0}, y_{1}, \cdots, y_{m}\right) \in \mathscr{R}^{2 m+2} \\
x_{\hbar}{ }^{-} \leq x_{\hbar} \leq x_{\hbar}{ }^{+}, y_{\hbar}{ }^{-} \leq y_{\hbar} \leq y_{\hbar}{ }^{+}, \hbar=0, \ldots, m .
\end{array}\right.
$$

and is based on the positivity of the coefficients of polynomial (7), for all possible combinations of uncertain parameters $p$. This choice is based on the well-known necessary condition for Hurwitz stability, that tends to produce a large search space. The advantage is that this space can be systematically obtained in a fast way from a set of linear inequalities, by solving a linear programming problem, and then, including the resulting region in a hyperrectangle, as describe in (16). More accurate search spaces could be obtained applying, for instance, the complete Routh-Hurwitz criterion [28], but at the price of more time-consuming and complex calculations to define the search space.

Since $\mathscr{C}$ defined as above can be a large search space, exhaustive grid techniques are usually unviable for a high resolution discretization. In this scenario, metaheuristics such as the PSO algorithm has proven to be useful, even for objective functions with discontinuities [30].

\section{Particle Swarm Optimization}

PSO is a bio-inspired algorithm proposed in [6], based on intelligent swarms, where the collective behavior of nonsophisticated agents creates global functional patterns, and it has already been used in control of power converters [7], [10][13].

In the sense of the design problem in this paper, the control gain vector (4) can be associated with the position of a particle $i$ in the search space, given by

$$
s_{i}=\left[x_{0 i}, x_{1 i}, \cdots, x_{m i}, y_{0 i}, y_{1 i}, \cdots, y_{m i}\right] .
$$

The swarm has a size of $N$ particles and must be sufficiently large to cover the search space.

The particles $i$ are randomly initialized on the search space $\mathscr{C}$, and in a given epoch $k$, for each particle position $s_{i}^{k}$, a real positive value is associated. This value is generated through the evaluation of the objective function (14), and it is called fitness. The particles positions in the search space are updated from one epoch $k$ to the next epoch $k+1$, until reaching the stop criterion, for instance, the limit of $M$ epochs for evolution. Each particle moves from the position $s_{i}^{k}$ to the next position $s_{i}^{k+1}$ with a velocity $v_{i}^{k}$, based on the equations

$$
\begin{gathered}
s_{i}^{k+1}=s_{i}^{k}+v_{i}^{k+1} . \\
v_{i}^{k+1}=\lambda v_{i}^{k}+\phi_{1} r_{1}\left(P_{i . b e s t}-s_{i}^{k}\right)+\phi_{2} r_{2}\left(G_{\text {best }}-s_{i}^{k}\right) .
\end{gathered}
$$

The velocity of a given particle is influenced by the best position that it got $\left(P_{i . b e s t}\right)$, and also by the best position among all particles $\left(G_{b e s t}\right)$. $\phi_{1}$ is the cognitive coefficient, $\phi_{2}$ is the social coefficient, $\lambda$ is the inertia factor and $r_{1}$ and $r_{2}$ are random values between $[0,1]$. It is also noted that the velocity of each particle, similarly to the position, is represented by a vector with the same dimension of (17) [6], [31].

The PSO algorithm used here can be summarized as follows:

a) configure the PSO parameters;

b) initialize the particles randomly in the search space;

c) calculate the objective function for each particle;

d) update $P_{i . b e s t}$ and $G_{\text {best }}$;

e) update position and velocity of each particle;

f) if the stop criteria is reached, continue. If not, increase the epoch number and return to $c$ );

g) return the best particle $\left(G_{\text {best }}\right)$ and end the execution.

The number of particles $N$, the number of epochs $M$, and the coefficients $\phi_{1}$ and $\phi_{2}$ are set in order to ensure convergence of the objective function with viable computational effort, and guidelines to setup these parameters can be found in [32], [33]. The algorithm stop criterion can be based on reaching $M$ or on stalling of the objective function. For instance, using MATLAB, this algorithm can be executed using the particleswarm function.

\section{Summary of the Proposed Procedure}

The proposed design procedure, encompassing the features presented in the previous sections, is summarized by the flowchart depicted in Figure 1.

The first step is to inform the plant model and the uncertain parameters, as shown in (1) and (2). In the sequence, the structure of the controller is chosen, defining coefficients vector (4). Then, the specifications in (11) and (12) are chosen by the control designer for evaluation of the objective function (14). The next step is to determine the search space (16), based on the closed-loop characteristic polynomial (7). Then, the setup parameters of the PSO are chosen and the optimization is carried out. In this step, it is worth to mention that, in each iteration of the PSO, each particle (candidate controller) is evaluated based on the objective function (14), including the assessment of robust stability using Kharitonov's Theorem.

The PSO algorithm described in Subsection 3.C runs until reaching the stop criterion. If the algorithm converges to a controller $c^{\star}$ (best particle of the swarm) for which the closedloop system is not KT stable, the procedure must be executed again, relaxing the limits of the uncertain plant parameters. Finally, if the algorithm converges to a controller $c^{\star}$, for which the closed-loop robust stability is successfully accessed by the Kharitonov's Theorem, and the constraints in (12) are satisfied, the procedure ends, providing $c^{\star}$ as a viable robust 


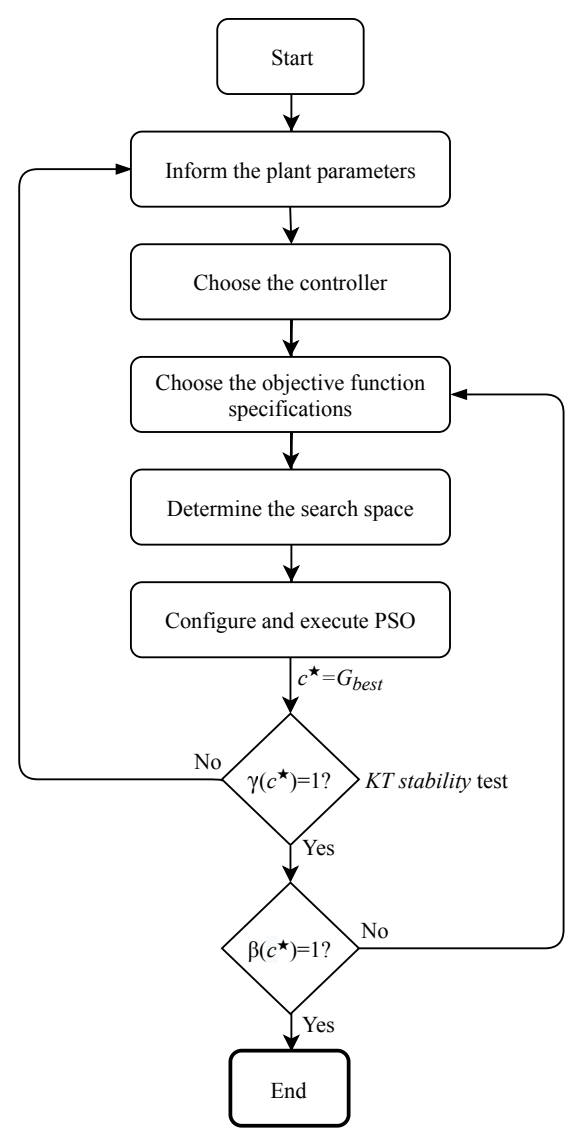

Fig. 1. Flowchart of the proposed design procedure.

controller. If the algorithm converges to a KT stable controller, but the constraints in (12) are not satisfied, the objective function specifications must be relaxed, and the procedure executed again.

It is worth to emphasize that the contribution here is the combination of the PSO and a novel objective function, including the Kharitonov's Theorem. Therefore, the proposed procedure can cope with multiple practical design constraints, leading to fixed control gains that ensure operation under limited control signal and include a theoretical certificate of stability under uncertain plant parameters.

In order to illustrate the effectiveness of the proposed procedure in the design of robust controllers for plants relevant in power electronics, in the next section, a case study is presented, given by the speed control of permanent magnet synchronous motor, with uncertain electrical and mechanical parameters.

\section{CASE STUDY: PERMANENT MAGNET SYNCHRONOUS MOTOR}

For an internal PMSM, consider a rotor field oriented control (FOC) strategy [34]. The FOC methods are usually employed to ensure high performance of the drive. This method results in a cascade control structure with two inner current control loops and an outer speed control loop, as given in Figure $2 a$. This structure decouples torque and flux using the $d q$ reference frame. The speed compensator generates the current reference $i_{q}^{*}$ processing the error between the speed reference $\omega_{m}^{*}$ and the actual speed $\omega_{m}$.

In order to implement a linear torque control, the constant torque angle strategy is adopted. Thus, $i_{d}^{*}=0$, and the torque generated depends only on the $i_{q}^{*}$ current component. For the current control loops, the phase currents $i_{a b c}$ are measured by Hall effect sensors, and then, based on the angular position $\theta_{r}$, are converted to the synchronous reference frame, using the Park transformations [35]. From the error between these currents and their references, the controllers $\mathrm{PI}_{d}$ and $\mathrm{PI}_{q}$ provide the voltages $v_{d}$ and $v_{q}$, which are then transformed to $a b c$ voltages. To drive the PMSM, these voltages are converted to pulse-width modulation (PWM) signals.

The model of the PMSM in synchronous reference frame is given by [36]

$$
\begin{aligned}
& \frac{d i_{d}}{d t}=-\frac{R_{s}}{L_{d}} i_{d}+\frac{L_{q}}{L_{d}} \omega_{e} i_{q}+\frac{1}{L_{d}} v_{d} \\
& \frac{d i_{q}}{d t}=-\frac{R_{s}}{L_{q}} i_{q}-\frac{L_{d}}{L_{q}} \omega_{e} i_{d}-\frac{\phi_{s r m}}{L_{q}} \omega_{e}+\frac{1}{L_{q}} v_{q}
\end{aligned}
$$

where the subscription $d$ and $q$ indicate the direct and quadrature axes. The stator inductances referred to the synchronous reference frame are given by $L_{d}$ and $L_{q}$, with different values for each axis due to the internal magnet motor configuration. The stator resistance is given by $R_{s}, \phi_{s r m}$ is the magnetic flux of the permanent magnet and $\omega_{e}$ is the electrical angular speed.

From (20) and (21), considering the terms depending on $\omega_{e}$ as disturbances, the following decoupled linear transfer functions can be obtained

$$
G_{d}(s)=\frac{I_{d}(s)}{V_{d}(s)}=\frac{\frac{1}{L_{d}}}{s+\frac{R_{s}}{L_{d}}}, \quad G_{q}(s)=\frac{I_{q}(s)}{V_{q}(s)}=\frac{\frac{1}{L_{q}}}{s+\frac{R_{s}}{L_{q}}} .
$$

The mechanical behavior can be described by the following dynamic model [36]

$$
\frac{d w_{m}}{d t}=\frac{1}{J}\left(\tau_{m}-B w_{m}\right)+\delta\left(w_{m}\right) .
$$

where $J$ represents the inertia of the rotor, $B$ is the viscous friction coefficient, $\delta\left(w_{m}\right)$ is a nonlinear term of bounded norm, and $\tau_{m}$ is the difference between the electromechanical torque, given by $\frac{3}{2} P\left[\left(L_{d}-L_{q}\right) i_{d}+\phi_{s r m}\right] i_{q}$, and the load torque. $P$ is the number of pairs of poles of the motor.

Assuming the mechanical torque as an input and the rotor speed as an output, the transfer function from the linear part of (23) is written as

$$
G_{n}(s)=\frac{W_{m}(s)}{T_{m}(s)}=\frac{\frac{1}{J}}{s+\frac{B}{J}} .
$$

The system parameters for this case study are summarized in Table I, including the uncertainties on the electrical and mechanical parameters. It is important to mention that, the interval of uncertainties must be provided by the control designer and is given, in this case study, from the expected uncertainty around the PMSM rated parameters. Nevertheless, the proposed procedure can handle with different intervals.

Note that (22) and (24) are first order linear plant models as presented in (1) that, based on the rated parameters shown in Table I, can be used to describe a PMSM subject to uncertain 


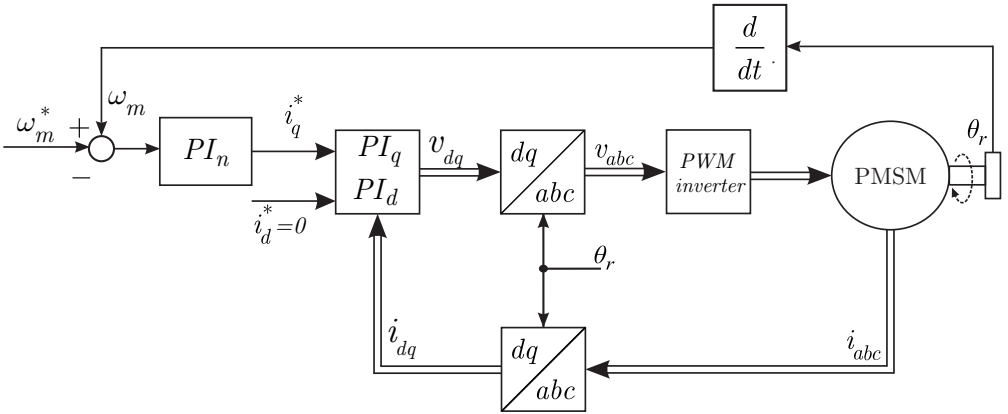

(a)

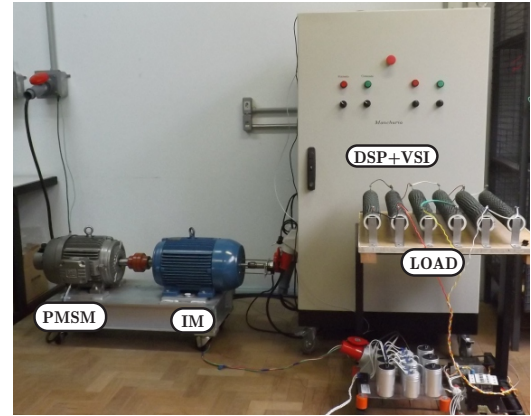

(b)

Fig. 2. Permanent magnet synchronous motor: (a) block diagram of the control system, comprised by three PI controllers; (b) prototype.

\section{TABLE I \\ PMSM Parameters}

\begin{tabular}{cc}
\hline Parameter & Value \\
\hline Rated Power & $11 \mathrm{~kW}$ \\
Rated Current & $19.2 \mathrm{~A}$ \\
Rated Torque & $58.4 \mathrm{Nm}$ \\
Poles $(\mathrm{P})$ & 6 \\
Stator Resistance $\left(R_{s}\right)$ & $0.475 \Omega \pm 40 \%$ \\
Inductance of d-axis $\left(L_{d}\right)$ & $20.1 \mathrm{mH} \pm 10 \%$ \\
Inductance of q-axis $\left(L_{q}\right)$ & $40.9 \mathrm{mH} \pm 10 \%$ \\
Rotor Inertia $(J)$ & $0.03877 \mathrm{kgm}{ }^{2} \pm 10 \%$ \\
Friction Coefficient $(B)$ & $0.0194 \mathrm{Nms} \pm 40 \%$ \\
PM flux linkage & $0.5126 \mathrm{~V} / \mathrm{rad} / \mathrm{s}$ \\
\hline
\end{tabular}

parameters, given, respectively, by

$$
G_{d}(s)=\frac{\frac{1}{L_{d}}}{s+\frac{R_{s}}{L_{d}}}=\frac{f_{0}\left(p_{1}\right)}{s+g_{0}\left(p_{1}, p_{2}\right)} .
$$

where $p_{1}=L_{d}, p_{2}=R_{s}, f_{0}=\frac{1}{L_{d}}$ and $g_{0}=\frac{R_{s}}{L_{d}}$,

$$
G_{q}(s)=\frac{\frac{1}{L_{q}}}{s+\frac{R_{s}}{L_{q}}}=\frac{f_{0}\left(p_{1}\right)}{s+g_{0}\left(p_{1}, p_{2}\right)} .
$$

where $p_{1}=L_{q}, p_{2}=R_{s}, f_{0}=\frac{1}{L_{q}}$ and $g_{0}=\frac{R_{s}}{L_{q}}$,

$$
G_{n}(s)=\frac{\frac{1}{J}}{s+\frac{B}{J}}=\frac{f_{0}\left(p_{1}\right)}{s+g_{0}\left(p_{1}, p_{2}\right)} .
$$

where $p_{1}=J, p_{2}=B, f_{0}=\frac{1}{J}$ and $g_{0}=\frac{B}{J}$.

Once the plant parameters are defined, the next step is to choose the controller structure. In this context, the control problem to be solved for this case study is to synthesize PI controllers with fixed gains for three control loops ( $d$ axis current, $q$-axis current and mechanical speed) ensuring stability and proper dynamic performance for the entire set of parameters given in Table I.

The third step in the proposed procedure is to choose the objective function specifications. For the mechanical speed plant, the reference values for system performance and stability margins are specified as

$$
\begin{array}{lll}
\omega_{c o}{ }^{*}=60 \mathrm{rad} / \mathrm{s} & \mathrm{PM}^{*}=60^{\circ} & \mathrm{GM}=5(14 d B) . \\
\overline{\mathrm{OV}}=10 \% & \overline{e_{s s}}=1 \% & \bar{u}=1
\end{array}
$$

Following the procedure, a two-dimensional search space for the gains $K_{P}\left(x_{1}\right)$ and $K_{I}\left(x_{0}\right)$ is defined based on the positivity of the coefficients of the characteristic polynomial (7). Therefore, considering the speed plant $G_{n}(s)$ in (27) and a generic PI controller, the closed-loop characteristic polynomial is given by

$$
D(s)=d_{2} s^{2}+d_{1} s+d_{0}=s^{2}+\left(\frac{B}{J}+\frac{1}{J} K_{P n}\right) s+\left(\frac{1}{J} K_{I n}\right) .
$$

where the inequalities that define the search space are given by

$$
K_{I n}>0 \quad K_{P n}>-B .
$$

The value of $10^{4}$ is chosen as an upper bound for $K_{P}$ and $K_{I}$ to have a large region for searching the control gains.

The fifth step in the design procedure is to setup the PSO parameters. Here, after some trial, one configuration that lead to good results was

$$
\mathrm{N}=200 \text { particles } \mathrm{M}=50 \text { epochs } \phi_{1}=0.5 \quad \phi_{2}=0.5 \text {. }
$$

The algorithm was executed several times, all of them in an offline way and independent from each other, leading to viable controllers. A typical execution for the speed control loop provided the PI control gains

$$
c_{n}^{\star}=\left[K_{I n} K_{P n}\right]=\left[\begin{array}{ll}
4.0169356855 & 0.9814291921
\end{array}\right] .
$$

For this execution, the evolution of the objective function over the epochs is given in Figure $3 a$, from which can be concluded that $\beta\left(c^{\star}\right)=1$ (indicating compliance with bounds in (12)) and $\gamma\left(c^{\star}\right)=1$ (indicating the KT stability). Figure $3 b$ shows the evaluation of the objective function for different values of $K_{P n}$ and $K_{I n}$, for a fine grid in the search space. Figure $3 c$ is the top view of Figure $3 b$, where the minimum region is evidenced. It is important to note that the region of the Figure $3 b$ was depicted with 10000 points and corresponds to about $0.01 \%$ of the search space area investigated by the PSO, requiring about 2 hours to be obtained with this exhaustive gridding. In this sense, the search provided by the PSO in 9 minutes is advantageous, justifying the choice of the algorithm for large search spaces.

To validate the results, the speed control loop was simulated with the control gains in (32). The frequency response of the open-loop transfer function $G_{c}(s) G_{n}(s)$ is shown in Figure $4 a$, for the vertices of the parametric uncertainty range given 


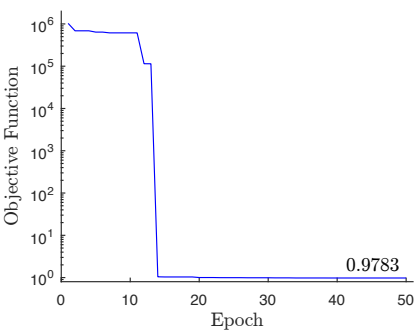

(a)

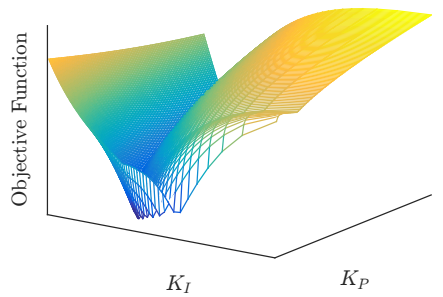

(b)

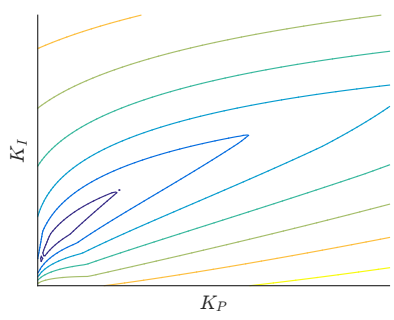

(c)

Fig. 3. Speed control loop: (a) fitness value on each epoch; (b) part of the region in the search space that involves the provided gain; (c) contour plot of (b).

in Table I. The worst case values of phase margin and crossover frequency were given by $\mathrm{PM}_{\min }=80.6^{\circ}, \omega_{\text {comin }}=$ $23.12 \mathrm{rad} / \mathrm{s}$. The control signal for the vertices of the system with gains (32) are shown in Figure $4 b$, where it is possible to confirm the compliance with the maximum value of the control signal, given in (28). The step responses of the closed-loop system for the vertices are shown in Figure $4 c$-top, from which it is possible to verify compliance with the design constraints.

In order to have a comparison between the gains obtained with the proposed procedure and the ones obtained with another tuning technique, Figure $4 c$-bottom shows the responses of a PI controller designed using pidtune, from MATLAB. In this case, the controller was designed for the minimum values of the coefficients in Table I, and considering the same specifications used for the PSO: $\omega_{c o}{ }^{*}=60 \mathrm{rad} / \mathrm{s}$ and $\mathrm{PM}^{*}=60^{\circ}$. Comparing the results, it is possible to verify superior performance of the closed-loop system with the PSObased PI controller, which presented a lower overshoot and a faster settling time. A more detailed performance comparison between the responses with these controllers will be presented in Section VI.

The proposed procedure is repeated for the control design of $d$-axis and $q$-axis current plants. For these, the reference values for crossover frequency is specified as $\omega_{c o}{ }^{*}=400 \mathrm{rad} / \mathrm{s}$

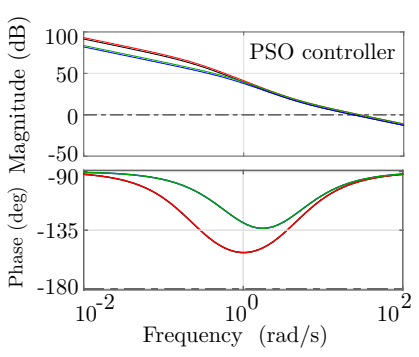

(a)

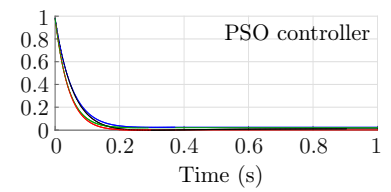

(b)

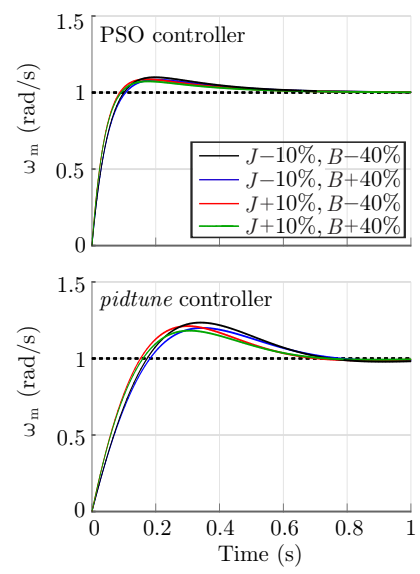

(c)
Fig. 4. Simulation results for the system (27): (a) Bode diagrams with gains (32); (b) control signal with gains (32); (c) Closed-loop step responses with gains (32) (top) and with pidtune gains (bottom). and the bound for control signal saturation is specified as $\bar{u}=17$. This value is one limit that ensures the operation of the closed-loop system without control saturation. The other specifications for system performance and stability are assumed as the same as in (28) and the PSO configuration the same as in (31). For the current plants $G_{d}(s)$ and $G_{q}(s)$, the procedure provided, respectively, the control gains

$$
\begin{gathered}
c_{d}^{\star}=\left[\begin{array}{ll}
K_{I d} K_{P d}
\end{array}\right]=\left[\begin{array}{ll}
508.3281745213 & 7.8272985293
\end{array}\right] . \\
c_{q}^{\star}=\left[K_{I q} K_{P q}\right]=\left[\begin{array}{ll}
1001.4258263209 & 15.9945084426
\end{array}\right] .
\end{gathered}
$$

\section{EXPERIMENTAL RESULTS}

To obtain the experimental results, a platform based on a commercial PMSM (WEG's WMagnet), with parameters described in Table I, was used. The digital signal processor (DSP) model TMS320F28335, from Texas Instruments, is used to digitally implement the controllers. The three-phase voltage source inverter (VSI) is based on IGBT switches. A $10 \mathrm{~kW}(500 \mathrm{~V}-20 \mathrm{~A})$ DC power supply is used on the DC bus. Hall effect sensors LV 25-P and LA 55$\mathrm{P}$, manufactured by LEM, are used for voltage and current measurements, respectively. An absolute encoder TRDNA256NWD provides the actual rotor position. A geometric PWM modulation is used [37]. The switching frequency used was $10 \mathrm{kHz}$, the sampling period is $100 \mathrm{~ms}$, and the PI controllers are discretized using Tustin method. Figure $2 b$ shows the experimental platform, where an induction machine (IM) is coupled to the axis of the PMSM. The electrical load shown in Figure $2 b$ is connected to the IM to produce a mechanical load disturbance of approximately $25 \mathrm{Nm}$ for the PMSM.

Figure 5 shows the experimental results for a reference tracking test, with the robust PSO-based PI controllers and also with the PI controllers designed using pidtune. Figure $5 a$-top shows the system start-up in ramp, followed by variations of speed setpoint. These variations are better detailed in Figure 5a-bottom, where is possible to notice a good tracking of the reference, with superior dynamic performance of the PSO-based PI controller. Figure $5 b$ top shows the $d$-axis current responses, while Figure $5 b$ bottom shows the $q$-axis current responses, highlighting the superior performance of the PSO-based controllers in all control loops. The deterioration of performance in practice for the pidtune controllers, are due the interaction between 
the three control loops, which are all affected by uncertain parameters, reinforcing one advantage of the robust controllers designed by means of the proposed procedure.

Figure 6 shows experimental results for rejection of a mechanical load disturbance, imposed by means of the IM coupled to the PMSM axis, as shown in Figure $2 b$. The system starts with a ramp of speed reference and, after reaching the setpoint (110 rad/s), step load disturbances of approximately $25 \mathrm{Nm}$ are applied. Figure $6 a$-top shows the speed responses, with transients zoomed in Figure $6 a$-bottom. It is possible to notice good disturbance rejection with the PSO-based PI controllers, with a superior performance when compared to the controllers obtained with the pidtune. Figure $6 b$-top shows the $d$-axis current responses, while Figure $6 b$-bottom shows the $q$ axis current responses, highlighting the superior performance of the PSO-based controllers in all control loops.

Finally, Figure 7 shows a comparison between the experimental and the simulation responses obtained for the four vertices of the with the PSO-based controllers. One can confirm the good correspondence between the average behavior of the experimental results and the simulations, indicating the suitability of the simple models to guide the proposed procedure for this application. The oscillations observed in the experimental results are due to mechanical vibrations in the coupling between the PMSM shaft and the encoder, as well as due to the nonideal construction of the machine, which produces torque oscillations.

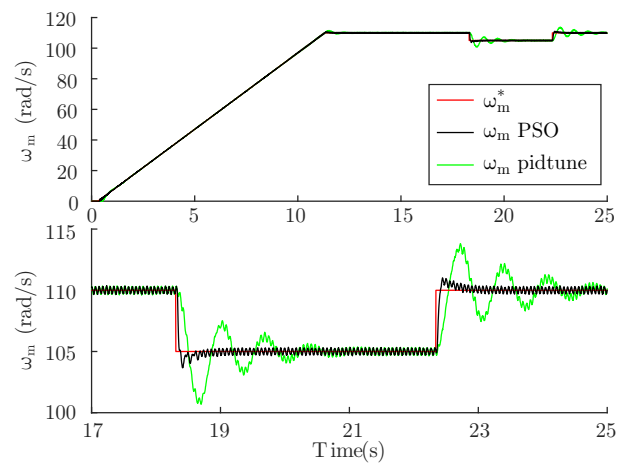

(a)

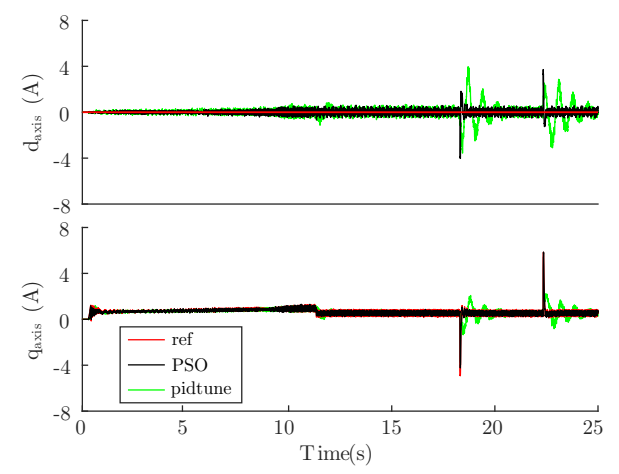

(b)

Fig. 5. Experimental results for reference tracking with the PI controllers under speed reference variations: (a) speed control loop responses (top) with detailed transients (bottom); (b) current control loop responses in $d$-axis (top) and $q$-axis (bottom).

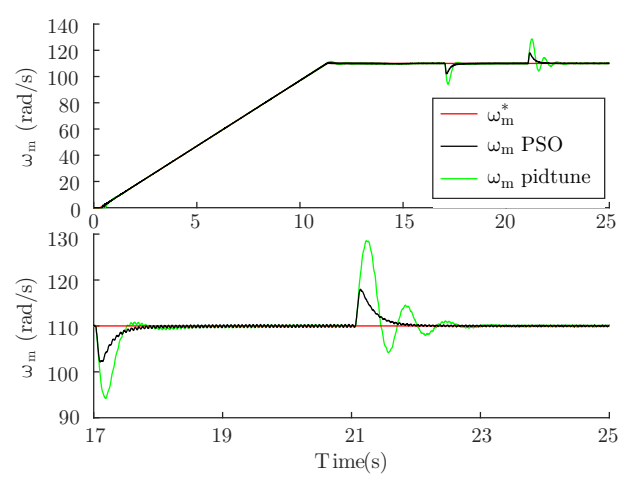

(a)

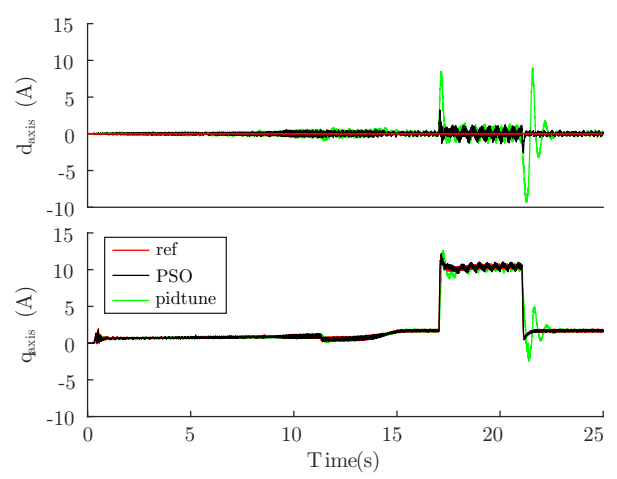

(b)

Fig. 6. Experimental results for reference tracking with the PI controllers under load variations: (a) speed control loop responses (top) with detailed transients (bottom); (b) current control loop responses in $d$-axis (top) and $q$-axis (bottom).

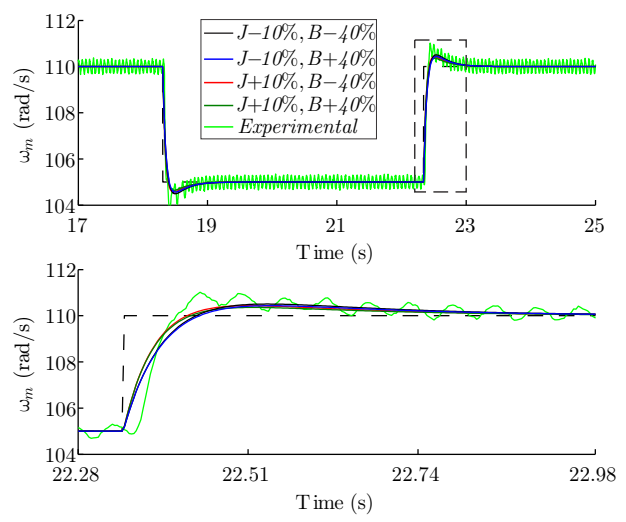

Fig. 7. Comparison between the simulations based on the MATLAB models for the four vertices of the polytope and the experimental result.

\section{COMPARATIVE ANALYSES}

This section presents a comparison between the controllers designed using the proposed procedure (called in this section, for simplicity, only as PSO) and controllers obtained by three other techniques.

For the three control loops, the comparisons are presented in Table II, where the measures are the worst case values for each design specification, obtained based on the responses for the vertices of the plant polytopic model.

Firstly, the responses with the PSO-based controllers are compared with the responses obtained by controllers designed 
TABLE II

Design Comparisons

\begin{tabular}{|c|c|c|c|c|c|c|c|c|c|c|c|c|}
\hline & \multicolumn{4}{|c|}{ Speed control loop } & \multicolumn{4}{|c|}{ Current control loop in $d$-axis } & \multicolumn{4}{|c|}{ Current control loop in $q$-axis } \\
\hline & PSO & pidtune & PSO-ITAE & GA & PSO & pidtune & PSO-ITAE & GA & PSO & pidtune & PSO-ITAE & GA \\
\hline PM (deg) & 80.6 & 60 & 90 & 82.7 & 81.6 & 60 & 90 & 89.1 & 81.04 & 60 & 90 & 84.15 \\
\hline$\omega_{c}(\mathrm{rad} / \mathrm{s})$ & 23.05 & 9.55 & $2.83 \times 10^{5}$ & 22.09 & 354.38 & 398.78 & $4.47 \times 10^{5}$ & 399.99 & 356.95 & 399.7 & $2.2 \times 10^{5}$ & 358.54 \\
\hline $\mathrm{GM}(\mathrm{dB})$ & $\infty$ & $\infty$ & $\infty$ & $\infty$ & $\infty$ & $\infty$ & $\infty$ & $\infty$ & $\infty$ & $\infty$ & $\infty$ & $\infty$ \\
\hline OV $(\%)$ & 9.96 & 23.4 & 0.0 & 8.14 & 8.44 & 23.3 & 0.0 & 0.0 & 9.42 & 23.8 & 0.0 & 6.71 \\
\hline$e_{s s}$ & zero & zero & zero & zero & zero & zero & zero & zero & zero & zero & zero & zero \\
\hline Saturation & no & no & yes & no & no & no & yes & no & no & no & yes & no \\
\hline KT stable & yes & no & no & yes & yes & yes & no & yes & yes & yes & no & yes \\
\hline$K_{P}$ & 0.981 & 0.351 & 9999.9 & 0.942 & 7.827 & 7.596 & 9999.9 & 8.966 & 15.99 & 15.6 & 9999.9 & 16.19 \\
\hline$K_{I}$ & 4.017 & 2.05 & 9980.2 & 2.894 & 508.3 & 1882.7 & 9988.9 & 169.3 & 1001.4 & 3732.6 & 9996.2 & 695.19 \\
\hline
\end{tabular}

using pidtune (same controllers used for the comparisons in Sections IV and V), which is a well-established control tuning function from MATLAB. In Table II, it can be noticed that the pidtune controllers lead to higher overshoot (as confirmed in Figure 4), due to the fact that the pidtune design is carried out based only in the phase margin and the crossover frequency. Moreover, the pidtune design consider only a nominal plant, and do not encompass a theoretical certificate of robust stability. Thus, the design may not ensure the $K T$ stability, as seen for the speed control loop.

The proposed PSO-based controllers are also compared with the execution of the PSO with an alternative objective function, the ITAE (integral time weighted absolute error) criterion, as presented in [29]. It can be noticed that the controllers provided by PSO-ITAE are not KT stable and lead to control saturation, since this method does not take into account these control input constraints in the design, optimizing only the time responses. Therefore, although the PSO-ITAE design may increase the crossover frequency, which leads to controllers with faster responses under unconstrained control signals, the practical implementation in power converters becomes infeasible due to bandwidth limitations. This conclusion reinforces the benefits of a suitable objective function encompassing multiple specifications, such as (14), in order to obtain controllers that can be implemented in practice.

Finally, the proposed PSO-based controllers are compared with designs where a genetic algorithm (GA), which is another well-established metaheuristic, is used to optimize the proposed objective function (14). The results with the PSO and the GA designs presented in Table II are very similar, for the speed and current control loops. Since both designs are based on the objective function (14), they ensure the $K T$ stability and do not exhibit saturation of the control signal. However, PSO leads to the control gains, in average, in half of the time demanded by the GA, for similar conditions (e.g., same number of particles for PSO and chromosomes for GA, epochs for PSO and generations for GA).

Moreover, defining success as a case where the algorithm leads to a controller $c^{\star}$ that has $\beta\left(c^{\star}\right)=1$ and $\gamma\left(c^{\star}\right)=1$, when repeating 20 times the execution of PSO and GA, the success rate with PSO is significantly higher than with the GA, as shown in Table III. In these 20 executions, the results with the PSO present a lower dispersion than the design based on the GA. The dispersion is defined as the standard deviation of the value of $f\left(c^{\star}\right)$ divided by its average value, as presented in Table III. These results confirm that the PSO is a suitable optimization algorithm for the proposed procedure.

TABLE III

Statistics Comparison between GA and PSO Algorithms for the Speed Control Loop

\begin{tabular}{c|cc}
\hline & PSO & GA \\
\hline Success rate & $95 \%$ & $40 \%$ \\
Dispersion & $1.29 \%$ & $4.52 \%$ \\
\hline
\end{tabular}

\section{CONCLUSIONS}

This paper proposed a design procedure for robust controllers applicable to power converters. The procedure is based on a PSO algorithm, that optimizes a multi-criteria objective function on the vertices of a polytope of plants and Kharitonov's Theorem that guarantees the closed-loop system is robustly stable for the entire domain of uncertainties. To cope with multiple practical design specifications in the proposed objective function can be a difficult task for known design tools, possibly leading to a time-consuming design stage, specially considering robustness against uncertain parameters and control signal limitation. This scenario reinforces the contribution of the paper, which is the combination of the PSO algorithm and the Kharithonov's Theorem in an automatic procedure for controllers design, including a robust stability certification. The proposed procedure was validated for an important application in power electronics, given by a PMSM speed controller, leading to viable controllers in practice. The comparisons demonstrate robustness under parametric uncertainties and confirm the good quality of the controllers, showing superiority over other control design methods. The proposed procedure is also applicable to high order plants and controllers, being an alternative that allows to reduce the trial and error stages for design of fixed gain controllers for power converters.

\section{ACKNOWLEDGMENTS}

This study was financed in part by the Coordenação de Aperfeiçoamento de Pessoal de Nível Superior - Brasil (CAPES/PROEX) - Finance Code 001. The authors also thank to INCT-GD, CNPq (465640/2014-1, 309536/2018-9 and 160884/2019-5), CAPES (23038.000776/2017-54), 
FAPERGS (17/2551- 0000517-1), and CAPES-PRINT (88887.465639/2019-00).

\section{REFERENCES}

[1] K. Deb, Multi-objective optimization using evolutionary algorithms, vol. 16, John Wiley \& Sons, July 2001.

[2] S. S. Rao, Engineering optimization: theory and practice, John Wiley \& Sons, June 2009.

[3] R. W. Erickson, Fundamentals of Power Electronics, Chapman \& Hall, New York, NY, 1997.

[4] S. P. Bhattacharyya, L. H. Keel, "Robust control: the parametric approach", in Advances in Control Education 1994, pp. 49-52, Elsevier, August 1994.

[5] R. Haupt, S. Haupt, Practical Genetic Algorithms, Wiley-Interscience publication, John Wiley, May 2004.

[6] R. Eberhart, J. Kennedy, "A new optimizer using particle swarm theory", in In Proceedings of the Sixth International Symposium on Micro Machine and Human Science., pp. 39-43, IEEE, Oct 1995.

[7] S. S. Sebtahmadi, H. B. Azad, S. H. A. Kaboli, M. D. Islam, S. Mekhilef, "A PSO-DQ current control scheme for performance enhancement of Z-source matrix converter to drive IM fed by abnormal voltage", IEEE Transactions on Power Electronics, vol. 33, no. 2, pp. 1666-1681, March 2017.

[8] R. Poli, J. Kennedy, T. Blackwell, "Particle swarm optimization: An Overview", Swarm intelligence, vol. 1, no. 1, pp. 33-57, August 2007.

[9] S. E. De León-Aldaco, H. Calleja, J. A. Alquicira, "Metaheuristic optimization methods applied to power converters: A review", IEEE Transactions on Power Electronics, vol. 30, no. 12, pp. 6791-6803, January 2015.

[10] M. Thirumeni, D. Thangavelusamy, "Design and analysis of hybrid PSO-GSA tuned PI and SMC controller for DC-DC Cuk converter", IET Circuits, Devices \& Systems, vol. 13, no. 3, pp. 374-384, January 2019.

[11] Z.-L. Gaing, "A particle swarm optimization approach for optimum design of PID controller in AVR system", IEEE transactions on energy conversion, vol. 19, no. 2, pp. 384-391, May 2004.

[12] R. A. Hanifah, S. F. Toha, S. Ahmad, M. K. Hassan, "Swarm-Intelligence Tuned Current Reduction for Power-Assisted Steering Control in Electric Vehicles", IEEE Transactions on Industrial Electronics, vol. 65, no. 9, pp. 7202-7210, December 2017.

[13] I.-Y. Chung, W. Liu, D. A. Cartes, E. G. Collins, S.-I. Moon, "Control methods of inverter-interfaced distributed generators in a microgrid system", IEEE Trans Ind Appl, vol. 46, no. 3, pp. 1078-1088, March 2010.

[14] J. Ackermann, Robust control: the parameter space approach, Springer Science \& Business Media, July 2012.
[15] V. L. Kharitonov, J. A. Torres-Muñoz, M. B. OrtizMoctezuma, "Polytopic families of quasi-polynomials: vertex-type stability conditions", IEEE Transactions on Circuits and Systems I: Fundamental Theory and Applications, vol. 50, no. 11, pp. 1413-1420, November 2003.

[16] K. E. L. Marcillo, D. A. P. Guingla, W. Barra, R. L. P. De Medeiros, E. M. Rocha, D. A. V. Benavides, F. G. Nogueira, "Interval robust controller to minimize oscillations effects caused by constant power load in a DC multi-converter buck-buck system", IEEE Access, vol. 7, pp. 26324-26342, February 2019.

[17] N. M. Dehkordi, N. Sadati, M. Hamzeh, "Robust tuning of transient droop gains based on Kharitonov's stability theorem in droop-controlled microgrids", IET Generation, Transmission \& Distribution, vol. 12, no. 14, pp. 3495-3501, August 2018.

[18] K. E. Lucas-Marcillo, D. A. P. Guingla, W. Barra, R. L. P. De Medeiros, E. M. Rocha, D. A. VacaBenavides, S. J. R. Orellana, E. V. H. Muentes, "Novel robust methodology for controller design aiming to ensure DC microgrid stability under CPL power variation", IEEE Access, vol. 7, pp. 64206-64222, May 2019.

[19] A. J. S. J. Veronica, N. S. Kumar, F. Gonzalez-Longatt, "Robust PI controller design for frequency stabilisation in a hybrid microgrid system considering parameter uncertainties and communication time delay", IET Generation, Transmission \& Distribution, vol. 13, no. 14, pp. 3048-3056, July 2019.

[20] R. He, Q. Han, "Dynamics and stability of permanentmagnet synchronous motor", Mathematical Problems in Engineering, vol. 2017, June 2017.

[21] A. K. Yadav, P. Gaur, P. Saxena, "Robust stability analysis of PMSM with parametric uncertainty using Kharitonov Theorem", Journal of Electrical Systems, vol. 12, no. 2, pp. 258-277, June 2016.

[22] J. Jung, Y. Choi, V. Q. Leu, H. H. Choi, "Fuzzy PI-type current controllers for permanent magnet synchronous motors", IET Electric Power Applications, vol. 5, no. 1, pp. 143-152, February 2011.

[23] M. Sreejeth, M. Singh, P. Kumar, "Particle swarm optimisation in efficiency improvement of vector controlled surface mounted permanent magnet synchronous motor drive", IET Power Electronics, vol. 8, no. 5, pp. 760-769, April 2015.

[24] B. R. Barmish, "A generalization of Kharitonov's fourpolynomial concept for robust stability problems with linearly dependent coefficient perturbations", IEEE Transactions on Automatic Control, vol. 34, no. 2, pp. 157-165, February 1989.

[25] S. Buso, P. Mattavelli, Digital Control in Power Electronics, Morgan \& Claypool Publishers, 2006.

[26] R. Teodorescu, M. Liserre, P. Rodríguez, Grid Converters for Photovoltaic and Wind Power Systems, Wiley - IEEE, John Wiley \& Sons, February 2011.

[27] A. Karimi, H. Khatibi, R. Longchamp, "Robust control of polytopic systems by convex optimization", Automatica, vol. 43, no. 8, pp. 1395-1402, August 2007. 
[28] R. C. Dorf, R. H. Bishop, Modern control systems, Pearson, 2011.

[29] S. Banerjee, A. Ghosh, N. Rana, "An Improved Interleaved Boost Converter With PSO-Based Optimal Type-III Controller", IEEE Journal of Emerging and Selected Topics in Power Electronics, vol. 5, no. 1, pp. 323-337, March 2017.

[30] S. Sengupta, S. Basak, R. Peters, "Particle Swarm Optimization: A survey of historical and recent developments with hybridization perspectives", Machine Learning and Knowledge Extraction, vol. 1, no. 1, pp. 157-191, October 2018.

[31] M. Veerachary, A. R. Saxena, "Optimized power stage design of low source current ripple fourth-order boost DC-DC converter: A PSO approach", IEEE Transactions on Industrial Electronics, vol. 62, no. 3, pp. 1491-1502, March 2015.

[32] R. C. Eberhart, Y. Shi, "Particle swarm optimization: developments, applications and resources", in Proceedings of the 2001 congress on evolutionary computation (IEEE Cat. No. 01TH8546), vol. 1, pp. 81-86, IEEE, May 2001.

[33] Y. Shi, R. C. Eberhart, "Parameter selection in particle swarm optimization", in International conference on evolutionary programming, pp. 591-600, Springer, March 1998.

[34] P. Pillay, R. Krishnan, "Modeling, simulation, and analysis of permanent-magnet motor drives. I. The permanent-magnet synchronous motor drive", IEEE Transactions on Industry Applications, vol. 25, no. 2, pp. 265-273, Mar/Apr 1989.

[35] P. Krause, O. Wasynczuk, S. Sudhoff, Analysis of Electric Machinery and Drive Systems, second ed., Wiley-IEEE Press, United States of America, March 2002.

[36] R. Krishnan, Electric motor drives: modeling, analysis, and control, Prentice Hall, February 2001.

[37] M. J. Ryan, R. D. Lorenz, R. De Doncker, "Modeling of multileg sine-wave inverters: a geometric approach", IEEE Transactions on Industrial Electronics, vol. 46, no. 6, pp. 1183-1191, December 1999.

\section{BIOGRAPHIES}

Lucas Cielo Borin received the B.Sc. degree in computer engineering, in 2017, and the M.Sc. degree in electrical engineering, in 2020, from the Federal University of Santa Maria, Brazil, where he is currently working toward the D.Sc. degree in electrical engineering with the Power Electronics and Control Research Group. His research interests include: intelligent algorithms and control theory applications.

\begin{tabular}{lcccr} 
Caio Ruviaro Dantas Osório & \multicolumn{2}{c}{ received the } \\
B.Sc. and M.Sc. & degrees in electrical
\end{tabular} engineering in 2015 and 2017, respectively, from thehttps://www.overleaf.com/project/5ef66290717bba0001d63ff9 Federal University of Santa Maria, Brazil, where he is currently working toward the D.Sc. degree in electrical engineering, with the Power Electronics and Control Research Group. His research interests include control theory applications on power electronics, robust control and robust stability analysis.

Gustavo Guilherme Koch received the B.Sc., M.Sc. and D.Sc. degrees in electrical engineering in 2013, 2015 and 2019, respectively, from the Federal University of Santa Maria, Brazil, where he is currently working toward the posdoctorate with the Power Electronics and Control Research Group. His research interests include robust control, and control theory applications.

Thieli Smidt Gabbi received the B.Sc., M.Sc. and D.Sc. degrees in electrical engineering in 2013, 2015 and 2019, respectively, from the Federal University of Santa Maria, Brazil. She is currently a Professor in the Federal University of Rio Grande do Sul (UFRGS). Her research interests include control systems and electrical machine drives.

Ricardo Coração de Leão Fontoura de Oliveira received the B.Sc. degree in computer engineering from the Pontifical Catholic University of Parana in 2001 and the M.Sc. and $\mathrm{Ph} . \mathrm{D}$. degrees in electrical engineering from the University of Campinas (UNICAMP), Campinas, SP, Brazil, in 2003 and 2006, respectively. He is currently a Professor with the School of Electrical and Computer Engineering (FEEC/UNICAMP). His research interests include the development of numerical tools for stability analysis and the control design of uncertain linear and fuzzy systems.

Vinícius Foletto Montagner received the Master's degree in electrical engineering from the Federal University of Santa Maria, Santa Maria, Brazil, in 2000, and the Ph.D. degree in electrical engineering from the University of Campinas, Campinas, Brazil, in 2005. He is currently a Professor in the Federal University of Santa Maria, where he works with the Power Electronics and Control Research Group. His research interests include robust stability and control applied to power electronics. 